\title{
Enhancing the Resilience of Low Earth Orbit Remote Sensing Satellite Networks
}

\author{
Jian Yang, Senior Member, IEEE, Dezheng Li, Xiaofeng Jiang, Member, IEEE, \\ Shuangwu Chen, and Lajos Hanzo, Fellow, IEEE
}

\begin{abstract}
The Low earth orbit Remote Sensing (LRS) satellite network is envisioned as an essential component of bolstering space information applications, thus extending conventional ground information applications to the on-board space information applications. However, it inevitably faces grave challenges imposed by the dynamics of the satellite network's topology, by the intermittence of Inter-Satellite communication Links (ISLs) as well as by the mobility-induced satellite-access switching of mobile terminals. Hence, this is a very different networking landscape from that of the terrestrial Internet. Against these challenging problems, we propose a resilient networking architecture for LRS Satellite Networks (LRS-SNs), with special emphasis on their dynamic routing, resilient transmission and their intrinsic mobility. Specifically, path-quality aided and lifetimeaware dynamic routing is proposed for enhancing the routing against dynamic topology changes. Hop-by-hop data transmission is relied upon for providing transmission resilience against ISL intermittence. Furthermore, data caching is invoked for providing resilience against intermittent communications imposed by dynamic satellite access switching. We employ on semi-physical simulation platform for evaluating the achievable performance of the proposed resilient network architecture.
\end{abstract}

\section{INTRODUCTION}

$\mathbf{T}$ He LRS satellites are widely used in the fields of ocean observation, agricultural monitoring, disaster prevention and mitigation, as well as in urban governance. The LRS-SN is capable of supporting observation missions requiring the cooperation of multiple satellites with the aid of its global coverage, near-real-time operation. It also has the advantages of low inter-satellite delay and high bandwidth. Hence, it is envisioned as a fundamental component of bolstering Space Information Network (SIN) applications. In recent years, numerous countries started to attach importance to LRSSN, as exemplified by Russia's Gosudarevo Oko (known as Sovereign's Eye) and by the Horizon 2020 Operational Network of Individual Observation Nodes (ONION) project of the European Union [1], as well as by the Gaofen-1 to Gaofen6 of China and the Planet Labs sensing remote satellites group. However, the LRS-SN exhibits a time-varying topology, intermittent ISLs and limited on-board resources. Furthermore, in the complex space environment, the satellites and ISLs have

J. Yang, D. Li, S. Chen, and X. Jiang were with the Department of Automation, Univ. of Science \& Technology of China. E-mail:jianyang@ustc.edu.cn.

L. Hanzo is with the Department of Electronics and Computer Science, University of Southampton, SO17 1BJ, UK. E-mail:lh@ecs.soton.ac.uk.

L. Hanzo would like to acknowledge the financial support of the Engineering and Physical Sciences Research Council projects EP/Noo4558/1, EP/PO34284/1, COALESCE, of the Royal Society's Global Challenges Research Fund Grant as well as of the European Research Council's Advanced Fellow Grant QuantCom. to operate in the face of electromagnetic interference, solaractivity-dependent energy limitation, and device imperfections, which impose challenges on their routing and transmission solutions both between the satellites and users.

The Low Earth Orbit (LEO) satellite network's routing algorithms can be classified into two types, namely Virtual Topology-based Routing Algorithms (VTRA) [2], [3], [4] and Virtual Node-based Routing Algorithms (VNRA) [5], [6], [7]. Specifically, in the context of VTRA, the constellation period is sliced into time slots according to the periodicity of the satellite ephemeris. Within a given time slot, the network topology is supposed to remain constant, thus conventional static routing algorithms like the Open Shortest Path First (OSPF) technique can be employed for calculating the routing path for each time slot. This scheme relies on a priori knowledge of the ephemeris and operates reliably by exploiting the deterministic and periodical dynamics of the satellite network topology. However, such conjectural and virtual topology based routing is vulnerable to ISL interruptions and failures. In the context of VNRA, the satellite network is modelled as the one consisting of virtual nodes with fixed geographic coordinates. The satellite closest to the fixed geographic coordinates is mapped to a virtual node. Relying on the fixed topology of virtual nodes, the routing mitigates the satellite movement impact by separating the network layer from the satellite topology dynamics. However, it is challenging to maintain reliable endto-end data transmission between satellites, since the routing path in the context of VNRA is between two virtual nodes, rather than the pair of satellites.

Traditional remote sensing satellites only support satelliteto-ground transmission applying high-speed Digital Data Transmission (DDT) owing to the huge data volume of highresolution optical remote sensing images. With the deployment of the On-Board Processing (OBP), remote sensing images can be compressed into small files and transmitted to terminals via multi-hop paths. This makes it possible for LRS-SN to provide networked observation services. A traffic-light-based routing protocol relying on preliminary routing planning is proposed in [8] for non-geostationary satellite IP networks. By contrast, an end-to-end transport protocol is proposed in [9] for satellite IP networks, which adopts a prompt bootstrapping algorithm to increase the transmission rate at the beginning of the session. However, the aforementioned advances in the context of satellite-based IP networks rely on TCP-like end-to-end transmission, hence they remain vulnerable to the mobilityinduced intermittence of satellite networks.

In the LRS-SN, the receiver is not limited to a ground 
station, it may be constituted by mobile terminals equipped with a receiver antenna, including hand-held terminals, vehicles, ships and aircraft. The diverse variety and potentially high velocity of terminals as well as the increased flexibility requirements of networked earth observation services also impose challenges on the mobility management of LRSSN. Mobile IP solutions are adequate for solving the mobility management problems of satellite-based IP network by establishing tunnels between the home agent and external agent. However, apart from encountering the triangular routing problem, using Mobile IP will impose a high cost in terms of binding updates associated with the frequent handovers caused by the high mobility of terminals and satellites. The changes of terminal IP caused by handovers is also detrimental in terms of session continuity. The Proxy Mobile IPv6 (PMIPv6) solutions were improved on the basis of Mobile IP and they are capable of supporting terminal mobility within a limited range by establishing tunnels between a Local Mobility Anchor (LMA) and a Mobile Access Gateway (MAG), thus reducing the signaling overhead and the frequency of session interruptions. In [10], a PMIPv6-based solution was proposed for mobile multicast scenarios, where both the MAG and the LMA were employed at the ground stations. Nevertheless, it remains a challenge to deploy reliable ground stations in disaster areas and in the middle of the oceans. In [11], a handoverindependent mobility management scheme is proposed for LEO satellite networks, which relies on geographical location information to make the mobility management independent of handovers. The scheme is indeed inspiring but for highmobility terminals the binding updates caused by the changes of IP address still cannot be readily dispensed with. Additionally, all the aforementioned solutions focus on addressing the problem caused by the handovers, but the issue of transmission efficiency reduction incurred by the changes of IP address has not been satisfactorily solved at the time of writing.

In this article, we aim for proposing a resilient LRS-SN architecture exhibiting resilience both to dynamic topology changes, as well as to link intermittence and to terminal mobility. In the resilient LRS-SN advocated, we rely on three key techniques for enhancing the network's resilience. As a proof of concept, we also implement the proposed conceptual architecture and investigate its key techniques in a prototype-based on-board router for evaluating its feasibility and applicability.

This article is organized as follows. We discuss the challenges and our basic ideas for enhancing the resilience of the LRS-SN. Next, we highlight the LRS-SN architecture, and then we introduce three key techniques for boosting the resilience of our proposed architecture. Then we evaluate our integrated solution, followed by our conclusion.

\section{Challenges And Basic Ideas for Satellite NETWORKING}

The first challenge is imposed by the random dynamics of the satellite nodes. Constrained by the high power-requirement of remote sensing in the face of the limited battery capacity, LRS satellites may have to be charged even after a single

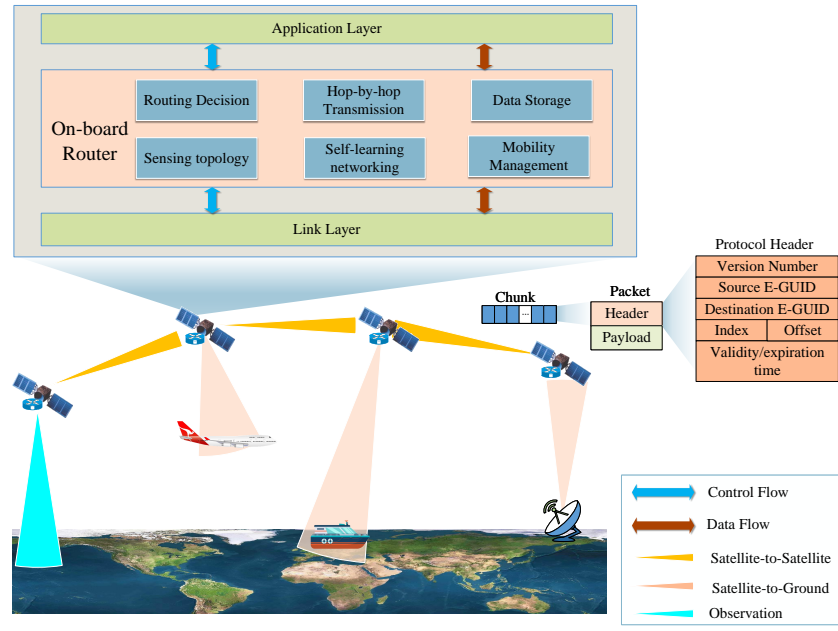

Fig. 1. Resilient LRS-SN architecture.

observation mission. The satellite in the state of being charged is unable to communicate with other satellites, which results in an undesirable topology change. The heavy inter-satellite communication and cooperative traffic may deplete the limited harvested energy in the battery and result in link failures, thus abruptly changing the network topology. The ISL communication is typically established by microwave/laser links which are susceptible both to Acquisition, Pointing and Tracking (APT) errors, as well as to Doppler shift and to random space noise. The link quality fluctuations may interrupt the inter-satellite communication and cause large delays, which imposed additional challenges on the static ISL-based routing mechanisms relying on the conjectural topology according to the ephemeris.

The second challenge is the intermittent nature of the satellite transmission. The traditional end-to-end transport protocol employs end-to-end acknowledgements and retransmissions for providing the upper layers with reliable data flow. This scheme is eminently suitable for wired networks since the communication links are reliable and the topology is fixed. By contrast, end-to-end acknowledgements and retransmissions result in low efficiency or even transmission failure because the packet losses encountered in the context of wireless satellite communications are frequent events, which imposed excessive end-to-end retransmissions.

The third challenge is the frequent access handover problem of mobile terminals. In the satellite IP network, the IP address represents both the network location and host identity. Mobile IP, which is proposed as a patch to support the mobility in IP networks, has to rely on a home agent for routing packets to the mobile node through a tunnel. Unfortunately, the frequent handovers encountered by the LRS-SN require frequent tunnel reconstruction, thus resulting in inefficient mobile IP operation. Furthermore, frequent handovers substantially aggravate the challenge of mobile registration and addressing, whilst additionally degrading the transmission efficiency owing to packet loss events during handovers, which has not been satisfactorily solved in the existing mobility management schemes of these IP networks. 
Motivated by the above challenges, we propose to incorporate sophisticated sensing, computing and caching capabilities into the routers of the satellite networks considered. The sensing capability is supported by periodically broadcasting messages to the neighbor on-board routers for evaluating the path quality and reachability. The sensing and computing capabilities allow us to conceive dynamic capable of prompt response to low ISL-quality or to ISL-failure events. The sensing and caching capability allow us to support resilient transmission capable of caching data for over-bridging of temporal link failures or packet losses, thus reducing the relative frequency of both end-to-end acknowledgements and retransmissions. By introducing the Equipment Global Unique Identifier (E-GUID) concept, we develop sophisticated intrinsic mobility management, which is capable of decoupling the host address and the identity, whilst additionally caching the data for supporting seamless data transmission, even in the face of frequent handovers.

\section{RESILIENT LRS-SN ARCHITECTURE}

Fig. II depicts the proposed LRS-SN, where each satellite has an on-board router, enabling on-orbit information delivery and sharing. In addition to ISLs, the LRS-SN also has satelliteto-ground links for the ground station and mobile terminals in order to access the space information infrastructure relying on the on-board equipment. Hence, there are three types of transmissions, i.e., satellite-to-satellite, satellite-to-ground and ground-to-ground via the satellite network.

We employ E-GUID instead of the IP for addressing nodes in the satellite networks while the terrestrial networks still apply the IP addressing. By relying on an appropriate protocol conversion between E-GUID and IP in the terrestrial gateway, the resilient LRS-SN architecture becomes compatible with the IP-based terrestrial networks. There are at least two main benefits of E-GUID addressing. Firstly, E-GUID remains unchanged, regardless of the associated logical or geographical location, which avoids the association updates imposed by the changes of IP address in the IP network. Secondly, due to the global uniqueness of the E-GUID, the satellite network remains capable of uniquely identifying each terminal even if a handover between access points occurs. This E-GUID based networking protocol assists us in solving the mobility management problem caused by the relatively high velocity of the satellites and terrestrial devices. The specific protocol packet header is characterized in Fig. II, which consists of the version number, source E-GUID, destination E-GUID, packet index and validity/expiration time. The top part of Fig. II portrays the six key modules of the on-board router:

- Topology Sensing: This module enables the on-board router to estimate the single/multi-hop path quality and lifetime by periodically broadcasting the space routing learning message (SRLM).

- Self-learning Networking: By analyzing the SRLMs received from other satellites, this module enables the on-board router to update the local routing table for supporting self-learning networking.

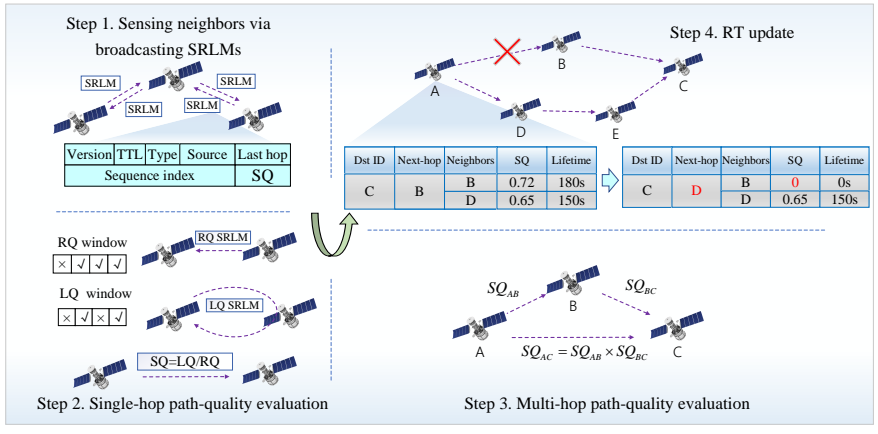

Fig. 2. Path-quality evaluation and routing update process of PLDR

- Routing Decision: This module interrogates the routing table and chooses the most appropriate forwarding rule to deliver data packets.

- Hop-by-hop Transmission: In contrast to the end-to-end transport protocol, this module allows the on-board router to transport the data in a hop-by-hop manner. Specifically, as shown in Fig. II, the transport unit is termed as a chunk consisting of multiple packets. Per-chunk selective acknowledgement, rather than per-packet acknowledgement, is employed for supporting reliable transport by retransmitting the packets that have been lost.

- Chunk Caching: In the context of hop-by-hop transmission, this module allows the router to temporarily store a chunk until it is successfully received by the next hop. In mobile scenarios, this caching module temporarily stores the chunks, while a terrestrial device is detached from the LRS-SN during the handover from one satellite to another for achieving seamless data transport.

- Mobility Management: This module is used for the mobility management of terrestrial devices. When a new device connects to the satellite or disconnects from the satellite, the on-board router notifies the LRS-SN using this module.

To address the challenges listed in Section II, we propose three key techniques, i.e., Path-quality aided and Lifetimeaware Dynamic Routing (PLDR) for coping with the random networking dynamics, which is implemented by relying on the combined action of the Routing Decision, Topology Sensing and Self-learning Networking modules, hop-by-hop based Resilient Transmission (RST) for guaranteeing data integrity, which is achieved by the concerted action of the Routing Decision, Hop-by-Hop Transmission as well as Chunk Caching, and data caching-aided intrinsic mobility management for mitigating the intermittent nature of the links owing to frequent handovers, which is supported with the aid of the Chunk Caching, Mobility Management and Self-learning Networking modules. The detailed implementation of the key technologies is discussed below.

\section{Key TeChNOLOGIES}

\section{A. Path-quality aided and lifetime-aware dynamic routing}

As a multi-hop wireless network, the LRS-SN supports mobile peer nodes, which is similar to the nature of the ad- 
hoc networks. The authors of [12] underline the advantages of using ad-hoc networking protocols in federated satellite systems through which near-real-time services can be supported by satellite networks. However, the existing routing protocols of ad-hoc networks, such as the Optimized Link State Routing (OLSR), consider only the link state for making routing decisions, without exploiting the predictability of node movement. By contrast, in our proposed Path-quality aided and Lifetime-aware Dynamic Routing (PLDR), the path lifetime, concept representing the duration of the ISL is introduced to assist in routing decisions. PLDR is a distributed routing protocol in which each node jointly determines the optimal next hop based on both path-quality and lifetime consideration. PLDR relies on sliding windows for evaluating the single-hop path-quality, thereby determining the multi-hop path-quality by forwarding the relevant path status messages, while the lifetime is calculated according to the satellite ephemeris information.

For sensing the real-time topology, each node periodically broadcasts SRLMs to its neighbor nodes, corresponding to Step 1 in Fig. 2. The SRLM comprises seven fields including the protocol version identifier, the type identifier, the source node E-GUID, the last-hop node E-GUID, the Time-To-Live (TTL), the sequence index, which ensures the timeliness of the SRLM and the path-quality all the way from the lasthop node to the source node, which is used for updating the Routing Table (RT) entries. The RT mainly records the routing information defining the path leading to the other nodes and the main fields of each entry are as follow:

- dst_id: E-GUID of the destination node.

- status: Boolean for marking the reachability of a node.

- last_srlm_seq: the latest sequence index for judging the timeliness of the received SRLM.

- neighbor_nodes: neighbors able to reach the destination.

- $s q$ : quality of the path to the destination.

- best_next_hop: the next hop of the optimal routing path.

A new RT entry is created once the node receives the SRLM generated by a new node, and the routing information of the node is updated according to the received SRLM.

Evaluating the path-qualities is the pivotal mechanism of dynamic routing. A pair of sliding windows, namely the Reception Quality (RQ) window and the Loop Quality (LQ) window are used for evaluating the single-hop path-quality, represented by Step 2 of Fig. 2. The RQ window counts the number of received SRLMs, which are generated and broadcast by the neighbor nodes, where the RQ value represents the success rate of the data transmission from the neighbor node to the local node. The system counts the number of the received SRLMs within the LQ window which are generated by the local node, rebroadcast to the local node by the neighbor nodes and broadcast in total twice. The LQ value represents the success rate of the data transmission of the loop spanning from the local node to its neighbor, and then back to the local node. The RQ window slides along with the maximal sequence index of the received RQ SRLMs for ensuring the timeliness of RQ. Furthermore, RQ will be FALSE if no RQ messages were received during the preset time limit. The LQ window slides along the time and LQ will be 0 if no LQ messages were received during the preset time limit. The bits in the sliding window which are corresponding to the sequence index of received RQ or LQ SRLMs would be marked in TRUE, followed by counting the number of marked bits in the LQ window. Both the RQ and LQ are calculated in accordance with the proportion of the number of the mark bits in the windows to the total window size. Then the Sending Quality (SQ) of the single-hop path, which represents the success rate of the data transmission from the local node to the neighbor node, can be calculated by $S Q=L Q / R Q$. The multi-hop path-SQ is then obtained by multiplying the single-hop path$\mathrm{SQ}$, corresponding to Step 3 in Fig. 2. The node compares the multi-hop path-SQ calculated and the maximal path-SQ recorded in the RT for determining whether the SRLM is from the optimal path and for updating the corresponding RT entries. To prevent the occurrence of SRLM flooding and routing loops, the nodes only broadcast the SRLMs from the optimal path, while dropping the others. The specific SRLM whose TTL is 0 will be dropped as well. In massive satellite networks, flooding can be further reduced with the aid of communication group partitioning and by limiting the propagation scope of SRLMs. The routes within each group are calculated via PLDR, while the routes leading to remote communication groups are calculated by the group agents.

For further improving the routing resilience, each single-hop path-lifetime is calculated for assisting the routing decisions. Once sensing a new neighbor node, the nodes exchange their Ephemeris Message (EM) with the neighbor. The EM consists of the satellite orbit parameters, which allows the node to sense the single-hop path-lifetime by calculating the specific moment of the neighbor moves beyond the communication range. After comparing the single-hop path-lifetime to the threshold, the specific paths having shorter duration than the threshold will not be considered in routing decisions, unless they constitute the only choice. The neighbor nodes for which the single-hop path-lifetime is higher than the threshold may then become the candidate next-hop towards the destination node. If the path-lifetime is lower than the threshold, the neighbor node information in the routing table will be deleted immediately, without waiting for the window to slide. As Step 4 of Fig. 2 shows, the nodes exploit the 'long path-lifetime' and 'optimal path-SQ' principles for finding the best next hop. They can also sense unpredictable satellite-failure or link-failure events and then adjust the routing path.

The convergence time of PLDR is defined as the time from the start of network topology changes to the instant, when all nodes have completed the updating of their routing table. To elaborate a little further, the convergence time includes the time required for sensing the single-hop path quality, the flooding time and the node processing time. The total time of the node processing and the flooding is less than $1 \mathrm{~s}$, which is negligible compared to the dozens of seconds required for single-hop path quality sensing. More explicitly, the time required for sensing the single-hop path quality is mainly affected by the size of the sliding windows and by the broadcast frequency of SRLMs. Generally, the sensing time of the single-hop path quality is approximately equal to 
the sliding window size multiplied by the SRLM broadcast period. Therefore, the nodes have a shorter sensing time at a more frequent SRLM broadcast and a shorter sliding window. SRLM broadcast frequency should be set by striking a compromise between the convergence time and the protocol overhead. Naturally, for different network scale and application requirements, different SRLM broadcast frequencies are suggested. The protocol overhead is essentially constituted by the signaling overhead generated by periodically broadcasting the SRLM, which generally only occupies a modest fraction of the ISL bandwidth.

\section{B. Hop-by-hop resilient data transmission}

RST is achieved by eliminating both the repeated/redundant traffic in the end-to-end path and by circumventing the typically low end-to-end transmission efficiency imposed by endto-end retransmission. Twin features of RST jointly increase the transmission resilience. Firstly, burst transmissions are used, where the data packets are aggregated into chunks which constitute the basic unit to be acknowledged and stored. By increasing the length of chunks, both the acknowledgement and storage efficiency is improved. Secondly, RST relies on hop-by-hop acknowledgements instead of end-toend acknowledgements, which maintains high transmission reliability, while eliminating repeated end-to-end traffic.

The specific process of RST is characterized in Fig. 3. At the beginning of transmission, a Chunk Synchronization Message (CSM) is sent to the receiver node to convey the chunkrelated side-information (Arrow 1), including the transmitter E-GUID, the receiver E-GUID, the chunk identifier and the total number of packets in this chunk. Then, the transmitter starts to send the data packets (Arrow 2) and an End of Chunk (EOC) message is sent after finishing the chunk transmission (Arrow 3). After receiving the EOC or after a timeout, the receiver notifies the transmitter of success or failure by an acknowledgement (ACK) flag (Arrow 4), which uses a bitmap for recoding the received packets of the chunk. The transmitter resends the lost packets requested by the ACK until the data has indeed been completely received (Arrow 5-7). During the whole transmission, the transmit node temporarily caches the data chunk until the chunk has been flawlessly received by receiving node, so that the data chunks are intact in each hop.

In case the link fails or the end-to-end path disappears, as shown in the right side of the Fig. 3, the remaining data chunks will be stored instead of being dropped, thus avoiding their retransmission from the source. The intermediate node will then continue to forward the remaining chunks once the path has been restored or a new path has been discovered via PLDR. Since the network layer has explicit knowledge about the final destination of each data chunk, it is capable of rerouting via a different path when a link failure occurs. This implies that the cached chunks are always routed to their targets before they become expired, which cannot be accomplished by conventional MAC-layer retransmission.

In the case of high path quality, there is no additional caching cost except for the data chunks being transmitted. However, in the case of low path quality, besides the chunks

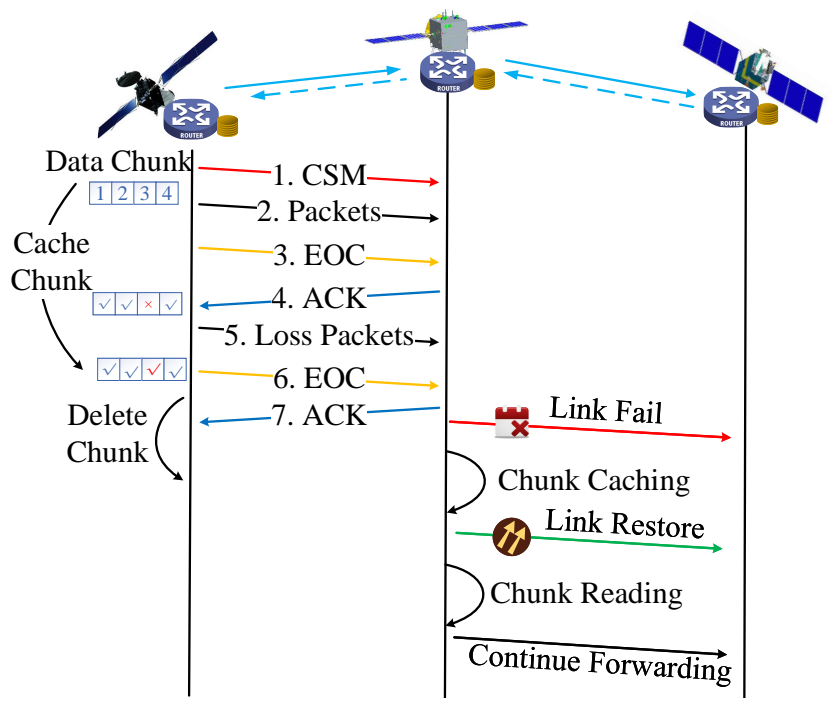

Fig. 3. Hop-by-hop resilient data transmission

waiting for acknowledgements, the pending data chunks stored in the nodes also occupy the storage resources, which may cause a high caching cost of RST. Generally, the caching cost may be deemed modest for on-board routers having GB level storage resources. Furthermore, since the power dissipation of the on-board router and of the inter-satellite communication unit is much lower than that of the remote sensing payload hardware, the total energy consumption of the onboard router and the inter-satellite communication unit, which are responsible for the information storage and signalling respectively, would not constitute a substantial burden for the energy resources of LRS satellites, even if their battery capacity is limited.

\section{Data caching aided intrinsic mobility management solution}

Data caching aided intrinsic mobility management is based on the E-GUID addressing, which plays a pivotal role in enhancing the intrinsic mobility of the LRS-SN.

The core task of the intrinsic mobility management is that of orchestrating the association between terminals and their access satellites, which is achieved by maintaining the Terminal Access Table (TAT). Specifically, each node maintains a TAT for storing the terminal access, and the TAT sets up an entry in the table for each reachable node. The TAT entry includes the satellite E-GUID, as well as the E-GUIDs of terminals connected to this satellite and the sequence number. To elaborate a little further, the sequence number indicates the time index of the TAT entry and will be increased by one, once the TAT entry changes. When a new terminal accesses the network, the direct-connection satellite will update its own TAT entry and notifies the other satellites through broadcasting the Terminal Access Messages (TAMs) containing its TAT. The TAM includes the direct-connection satellite E-GUID, the index of the TAT entry and the attached terminal E-GUIDs. After receiving the TAM, the satellite entry will be updated according to the TAM information if the entry index in the 


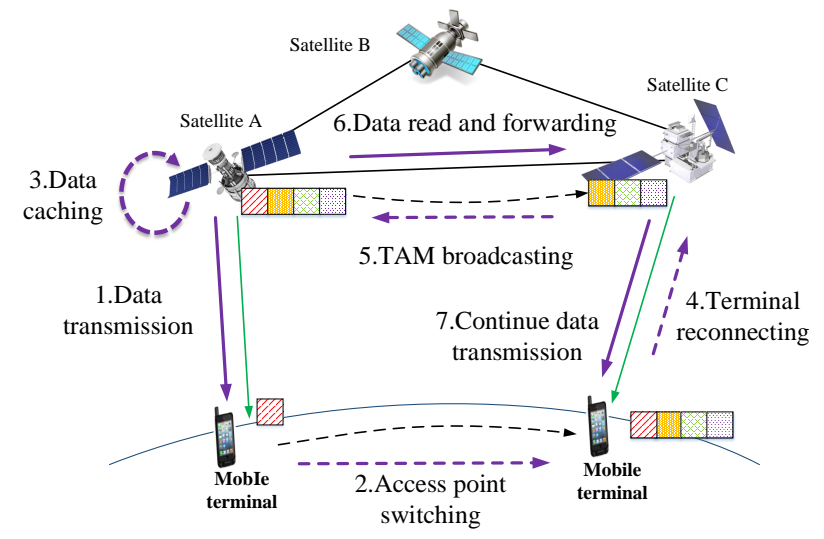

Fig. 4. Data caching aided intrinsic mobility solution

local TAT is smaller than the index in TAM. Otherwise, the TAM is dropped to prevent any potential message flooding.

As an indispensable function of the intrinsic mobility management, the intermittent nature of the LRS-SN is mitigated by a powerful combination of network device identity sensing and the cache capability of the nodes. Similar to inter-satellite transmissions, the intrinsic mobility management relies on hop-by-hop acknowledgements for ensuring a high transmission integrity by also exploiting the satellite-ground links. As shown in Fig. 4, when a handover caused by the relative movement between a terminal and a satellite occurs during the satellite-to-ground transmission (Arrow 1-2), the last directconnection satellite stores the undelivered data chunks (Arrow 3 ) and waits for the terminal to re-access to the network. The new direct-connection satellite updates the local TAT when the terminal regains access to the network (Arrow 4) and then sends the TAM for notifying all the other satellites (Arrow 5). Since the terminal globally E-GUID is unique, the last directconnection satellite is aware of the uncompleted transmission task of the terminal. Hence it reads the data chunks from the cache and continues forwarding the rest of data chunks to the new direct-connection satellite (Arrow 6-7).

\section{Performance Evaluations}

In order to quantify the performance of the proposed resilient LRS-SN, we have constructed a semi-physical simulation platform for emulating a satellite network and collected real data. The platform consists of two basic parts: the network controller, which configures the network in real time, and the LRS-SN satellites emulated by the Raspberry Pis (RPis) that implement the proposed conceptual architecture relying on our key techniques. The network controller calculates the communication distance between satellites through the Systems Tool Kit (STK) according to the satellite orbit information. Due to the lack of multi-path links and scatters in inter-satellite communication, only the free space path loss is considered for simplifying our simulations. The noise is assumed to be additive white Gaussian noise. The satellites communicate both with each other and with the controller through the socket interfaces.

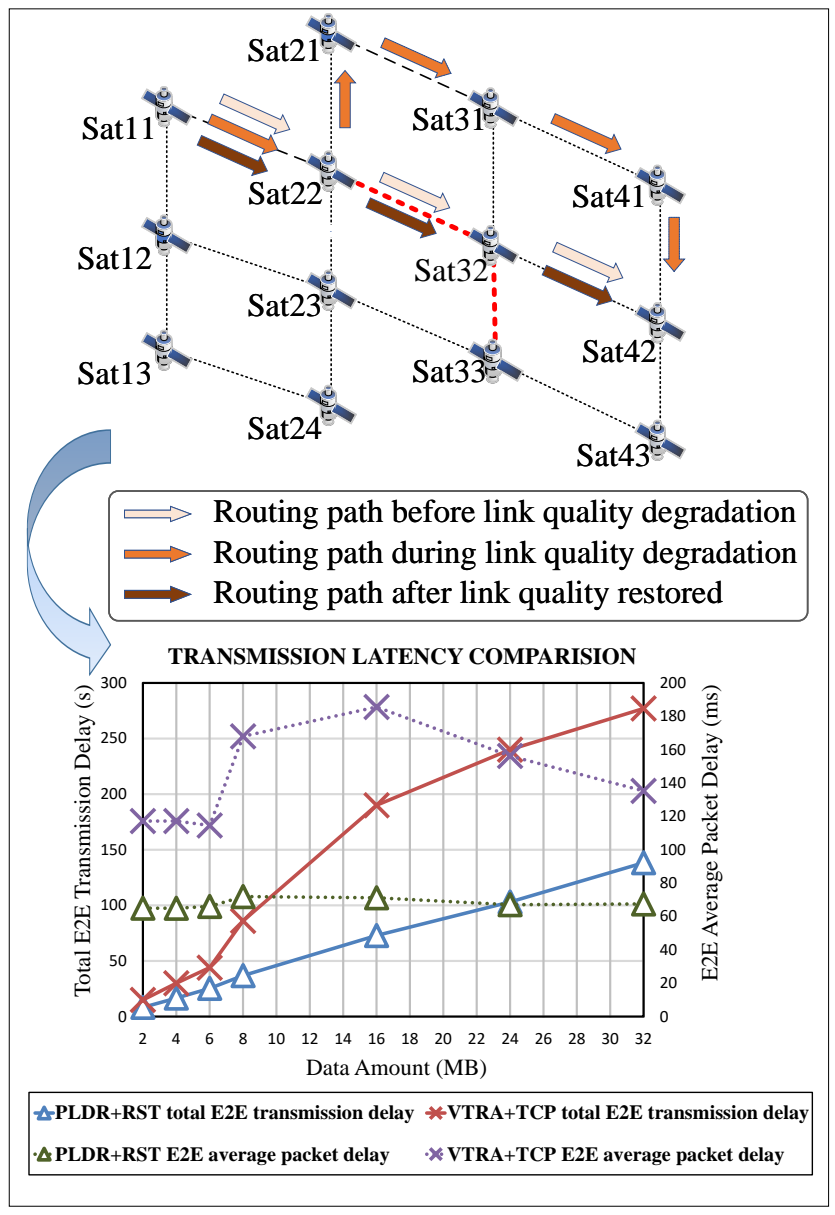

Fig. 5. Experimental configuration and the performance of dynamic routing as well as of resilient transmission

We set up a network topology having 13 satellite nodes in four different orbits, as shown in Fig. 5. The link qualities of the ISL Sat22 $\rightarrow$ Sat32 and ISL Sat32 $\rightarrow$ Sat33 were suddenly degraded at $t_{1}$ to simulate random events, and the qualities were restored at $t_{2}$. The experimental results of routing decisions, which are marked by different arrows in Fig. 5, show that the nodes are able to sense the path quality fluctuations and hence make more reasonable routing decision via PLDR. And we can also find that during the entire transmission, no packet loss events are inflicted, which indicates that perfect end-to-end data integrity is achieved. We also performed comparisons between the RST with PLDR and the conventional TCP with VTRA. The experimental results shown at the bottom of Fig. 5 indicate that for the task of delivering $30 \mathrm{MB}$ of data, the proposed technique significantly reduced the end-to-end latency and mitigated the link quality degradation by gracefully increasing the total end-to-end (E2E) transmission delay (left-hand axis), whilst maintaining a near-constant E2E average packet delay (righthand axis). To elaborate a little further, observe in Fig. 5 that TCP with VTRA requires almost twice the transmission time of the RST with PLDR at a 30 MB data-load, since the VTRA fails to sense the link quality degradation, hence degrading the E2E transmission efficiency. 


\section{CONCLUSION}

In this article, we have proposed an enhanced-resilience networking solution for LRS-SN operating in the face of dynamic topology changes imposed by both terminal and satellite mobility. We presented three key techniques for enhancing the network resilience against the aforementioned networking dynamics. These key techniques have been integrated into our semi-physical simulation platform for characterizing our resilient networking architecture designed for LRS-SN. Our further work will focus on extending our architecture to largescale LRS-SN having hundreds or even thousands of satellites with the aid of both clustering and multi-layer satellite network architectures [13].

\section{REFERENCES}

[1] E. Alarcn et al., "Design and Optimization of a Polar Satellite Mission to Complement the Copernicus System," IEEE Access, vol. 6, pp. 34777 34 789, 2018.

[2] H. S. Chang et al., "FSA-Based Link Assignment and Routing in LowEarth Orbit Satellite Networks," IEEE Trans. Veh. Technol., vol. 47, no. 3, pp. 1037-1048, Aug. 1998.

[3] Y. Lu et al., "Virtual Topology for LEO Satellite Networks Based on Earth-Fixed Footprint Mode," IEEE Commun. Lett., vol. 17, no. 2, pp. 357-360, Feb. 2013.

[4] T. Taleb et al., "Explicit Load Balancing Technique for NGEO Satellite IP Networks with On-Board Processing Capabilities," IEEE/ACM Trans. Netw., vol. 17, no. 1, pp. 281-293, Feb. 2009.

[5] E. Ekici et al., "A Distributed Routing Algorithm for Datagram Traffic in LEO Satellite Networks," IEEE/ACM Trans. Netw., vol. 9, no. 2, pp. 137-147, Apr. 2001.

[6] J. Li et al., "Temporal Netgrid Model based Dynamic Routing in LargeScale Small Satellite Networks," IEEE Trans. Veh. Technol., 2019, DOI: 10.1109/TVT.2019.2910570.

[7] Y. Yang et al., "Towards Energy-Efficient Routing in Satellite Networks," IEEE J. Sel. Areas Commun., vol. 34, no. 12, pp. 3869-3886, Dec. 2016.

[8] G. Song et al., "TLR: A Traffic-Light-Based Intelligent Routing Strategy for NGEO Satellite IP Networks," IEEE Trans. Wireless Commun. vol. 13, no. 6, pp. 3380-3393, Jun. 2014.

[9] L. Jiong et al., "TP-Satellite: A New Transport Protocol for Satellite IP Networks," IEEE Trans. Aerosp. Electron. Syst., vol. 45, no. 2, pp. 502-515, Apr. 2009.

[10] E. K. Jaff et al., "IP Multicast Receiver Mobility Support Using PMIPv6 in a Global Satellite Network," IEEE Commun. Mag., vol. 53, no. 3, pp. 30-37, Mar. 2015.

[11] H. Tsunoda et al., "Supporting IP/LEO Satellite Networks by HandoverIndependent IP Mobility Management," IEEE J. Sel. Areas Commun., vol. 22, no. 2, pp. 300-307, Feb. 2004.

[12] J. A. Ruiz-De-Aza et al., "Benefits of Using Mobile Ad-Hoc Network Protocols in Federated Satellite Systems for Polar Satellite Missions," IEEE Access, vol. 6, pp. 56356-56367, 2018.

[13] X. Huang, J. A. Zhang, R. P. Liu, Y. J. Guo, and L. Hanzo, "AirplaneAided Integrated Networking for 6G Wireless: Will It Work?" IEEE Veh. Technol. Mag., vol. 14, no. 3, pp. 84-91, Sep. 2019.

Jian Yang [SM'15] (jianyang@ustc.edu.cn) received his B.S. and Ph.D. degrees from University of Science and Technology of China (USTC). He is currently a professor in the School of Information Science and Technology, USTC. His research interests include future intelligent network, multimedia over wired \& wireless and stochastic optimization.

Dezheng Li (ldz001@mail.ustc.edu.cn) received the B.S. degrees from USTC. $\mathrm{He}$ is currently pursuing Ph.D. degree in USTC. His research interests include satellite networks and cross-layer network optimization.
Xiaofeng Jiang [M'13] (jxf@ustc.edu.cn) Xiaofeng Jiang received the B.E. and $\mathrm{Ph} . \mathrm{D}$. degrees from USTC, in 2008 and 2013, respectively. He is currently a research fellow in USTC. His recent research interests include spectrum sensing, discrete event dynamic system, and wireless communications.

Shuangwu Chen received the B.S. and Ph.D. degrees from USTC. He is currently a Post-Doctoral Researcher in USTC. His research interests include future intelligent network, multimedia over wired and wireless network and stochastic optimization.

Lajos Hanzo F'04 (http:www-mobile.ecs.soton.ac.uk, https:en.wikipedia.org/wiki/Lajos_Hanzo) FREng, FIET, Fellow of EURASIP, DSc received his Master degree in electronics in 1976 and his doctorate in 1983. He holds an honorary doctorate by the Technical University of Budapest (2009) and by the University of Edinburgh (2015). He is a Foreign Member of the Hungarian Academy of Sciences and a former Editor-in-Chief of the IEEE Press. He has served as Governor of both IEEE ComSoc and of VTS. He has published 1900+ contributions at IEEE Xplore, 18 Wiley-IEEE Press books and has helped the fast-track career of $119 \mathrm{PhD}$ students. 